\title{
Tea consumption and risk of diabetes in the Chinese population: a multi-centre, cross-sectional study
}

\author{
Yaling Chen ${ }^{1}$, Wei $\mathrm{Li}^{1}$, Shanhu Qiu ${ }^{1}$, Carvalho Vladmir ${ }^{1}$, Xiaohan Xu${ }^{1}$, Xinling Wang ${ }^{2}$, Xin Nian ${ }^{3}$, \\ Qingyun Chen ${ }^{4}$, Qing Wang ${ }^{5}$, Ping Tu ${ }^{6}$, Lihui Zhang ${ }^{7}$, Sunjie Yan ${ }^{8}$, Kaili Li ${ }^{9}$, Juan Chen ${ }^{1}$, Hang Wu ${ }^{1}$, \\ Xuyi Wang ${ }^{1}$, Xiaohang Wang ${ }^{1}$, Jingbao Liu ${ }^{1}$, Min Cai ${ }^{1}$, Zhiyao Wang ${ }^{10}$, Bei Wang ${ }^{11}$ and Zilin Sun ${ }^{1 *}$ \\ ${ }^{1}$ Department of Endocrinology, Zhongda Hospital, Institute of Diabetes, School of Medicine, Southeast University, Nanjing \\ 210009, People's Republic of China \\ ${ }^{2}$ Department of Endocrinology, People's Hospital of Xinjiang Uyghur Autonomous Region, Urumqi 830001, People's Republic of \\ China
}

${ }^{3}$ Department of Endocrinology, First Affiliated Hospital of Kunming Medical University, Kunming 650032, People's Republic of China

${ }^{4}$ Department of Endocrinology, The First Affiliated Hospital of Guangxi Medical University, Nanning 530021, People's Republic of China

${ }^{5}$ Department of Endocrinology, China-Japan Union Hospital of Jilin University, Changchun 130031, People's Republic of China ${ }^{6}$ Department of Endocrinology, The Third Hospital of Nanchang, Nanchang 330009, People's Republic of China

${ }^{7}$ Department of Endocrinology, The Second Hospital of Hebei Medical University, Shijiazhuang 050035, People's Republic of China ${ }^{8}$ Department of Endocrinology, The First Affiliated Hospital of Fujian Medical University, Diabetes Research Institute of Fujian Province, Fuzhou 350005, People's Republic of China

${ }^{9}$ Department of Endocrinology, Xinjiang Uygur Autonomous Region Hospital of Traditional Chinese Medicine, Urumqi 830006, People's Republic of China

${ }^{10}$ Suzhou MetroHealth Medical Technology Co. Ltd, Suzhou 215101, People's Republic of China

${ }^{11}$ School of Public Health, Southeast University, Nanjing 210009, People's Republic of China

(Submitted 25 July 2019 - Final revision received 16 October 2019 - Accepted 30 October 2019 - First published online 25 November 2019)

\section{Abstract}

The aim of the present study was to explore the influence of tea consumption on diabetes mellitus in the Chinese population. This multi-centre, cross-sectional study was conducted in eight sites from south, east, north, west and middle regions in China by enrolling 12017 subjects aged 20-70 years. Socio-demographic and general information was collected by a standardised questionnaire. A standard procedure was used to measure anthropometric characteristics and to obtain blood samples. The diagnosis of diabetes was determined using a standard 75-g oral glucose tolerance test. In the final analysis, 10825 participants were included and multiple logistic models and interaction effect analysis were applied for assessing the association between tea drinking with diabetes. Compared with non-tea drinkers, the multivariable-adjusted OR for newly diagnosed diabetes were $0 \cdot 80$ (95\% CI 0.67, 0.97), $0 \cdot 88$ (95\% CI $0 \cdot 71,1 \cdot 09$ ) and 0.86 (95\% CI 0.67, $1 \cdot 11$ ) for daily tea drinkers, occasional tea drinkers and seldom tea drinkers, respectively. Furthermore, drinking tea daily was related to decreased risk of diabetes in females by $32 \%$, elderly ( $>45$ years) by $24 \%$ and obese (BMI $>30 \mathrm{~kg} / \mathrm{m}^{2}$ ) by $34 \%$. Moreover, drinking dark tea was associated with reduced risk of diabetes by $45 \%$ (OR $0.55 ; 95 \% \mathrm{CI} 0 \cdot 42,0 \cdot 72 ; P<0 \cdot 01)$. The results imply that drinking tea daily was negatively related to risk of diabetes in female, elderly and obese people. In addition, drinking dark tea was associated with decreased risk of type 2 diabetes mellitus.

Key words: Tea consumption: Lifestyle: Diabetes mellitus: Risk

Diabetes mellitus is a complex metabolic syndrome and has been cited by the WHO as the third most serious non-communicable disease that threatens people's health, following cancer and CVD. It was reported that the prevalence of diabetes in China has reached a staggering $10.9 \%$; what is more, it was estimated that 388.1 million Chinese adults (200.4 million men and
187.7 million women) may have suffered from pre-diabetes in $2013^{(1)}$. Furthermore, according to the eighth IDF Diabetes Atlas in 2017, the cost of diabetes reached $\$ 727$ billion, of which China's medical expenses for diabetes reached $\$ 110$ billion $^{(2)}$. Former studies have demonstrated that diabetes is caused by the combination of genetic, environmental and modifiable

Abbreviation: T2DM, type 2 diabetes mellitus.

* Corresponding author: Zilin Sun, email sunzilin1963@126.com 
lifestyle factors which are considered extremely significant in the prevention of type 2 diabetes mellitus (T2DM) ${ }^{(3-5)}$.

After water, tea is the second most consumed beverages in many parts of the world and has been regularly consumed for centuries in China. Tea is generally divided into five categories based on how it is processed ${ }^{(6)}$ : unfermented tea (green tea), 10-20\% fermented tea (white tea), semi-fermented tea (oolong tea) and fully fermented tea (black tea and dark tea) ${ }^{(7)}$. Black tea is made by using endogenous enzymes (polyphenol oxidase) to promote fermentation, whereas dark tea is developed through a series of processes and fermented completely, also with the help of exogenous enzymes. Finally, the differences in the fermentation process may lead to different effects on diabetes with these diverse phytochemicals ${ }^{(8)}$.

The predominant bioactive substance contained in tea, for example, catechin, caffeine, thearubigins and polyphenols, has been suggested to have anti-diabetic effects in diabetic mice ${ }^{(9)}$. In addition, researches ${ }^{(10,11)}$ reported that tea consumption could prevent the development of obesity, T2DM and cancer. In recent years, population-based studies about association between tea consumption and diabetes have received significant attention. A survey conducted in Poland showed that tea consumption was negatively correlated with central obesity and fasting plasma glucose in adults ${ }^{(12)}$. The Singapore Chinese Health Study aged 45-74 years reported that consuming $\geq 1$ cup of black tea/d had a potential $14 \%$ reduction in risk of $\mathrm{T} 2 \mathrm{DM}$ (risk ratio $0 \cdot 86 ; 95 \%$ CI $0.74,1.00)$ compared with non-consumers and reported no association with green tea ${ }^{(13)}$. Nevertheless, studies in Japan found that oolong tea may increase the risk of T2DM in Japanese women adults, but there was no correlation of green tea and black tea with the risk of diabetes ${ }^{(14)}$. A cross-sectional study conducted in a community-based specialised care centre in Pakistan observed that higher consumption of tea was independently associated with uncontrolled T2DM, as OR 1.5 (95\% CI 1.0, 2.2 $)^{(15)}$. In short, inconsistent results have been reported in different subjects; it seems to be prudent for tea drinkers to acquire effective prevention. The aim of the present study was to investigate the connection of tea consumption with T2DM within a large representative sample in the Chinese population.

\section{Methods}

\section{Study design and population}

This multi-centre, cross-sectional study, based on the 'Non-lab and semi-lab algorithms for screening undiagnosed diabetes' study $^{(16)}$, was conducted in eight centres including seven provinces in China from November 2016 to June 2017. A multi-stage cluster and simple randomisation method was applied to recruit subjects aged $20-70$ years in 1-2 provinces that were randomly selected in east, south, west, north and central regions across China. Finally, a total of 12017 participants completed the investigation, including three parts: face-to-face questionnaire, physical examination and laboratory examination, in which fasting blood (at least $8 \mathrm{~h}$ ) in the morning and postprandial blood (after oral administration of $75 \mathrm{~g}$ glucose) were drawn.

After excluding 100 subjects with missing data on sociodemographic information (e.g. age, sex and nationality), 649 with self-reported diabetes, thirty-two with defined other nationality, 355 with missing important laboratory indexes (including fasting plasma glucose, 2 -h plasma glucose) or seven common lifestyle characteristics (such as diet habits, physical exercise, alcohol drinking, tea consumption and smoking status) and substituting the mean and the median for empty data (containing TAG, total cholesterol, HDL, LDL, waist circumference, height, weight, systolic blood pressure, diastolic blood pressure), outliers with waist circumference and $\mathrm{BMI}>95.5$ percentile or $<0.5$ percentile, a total of 10825 participants were included in the final data analysis (Fig. 1).

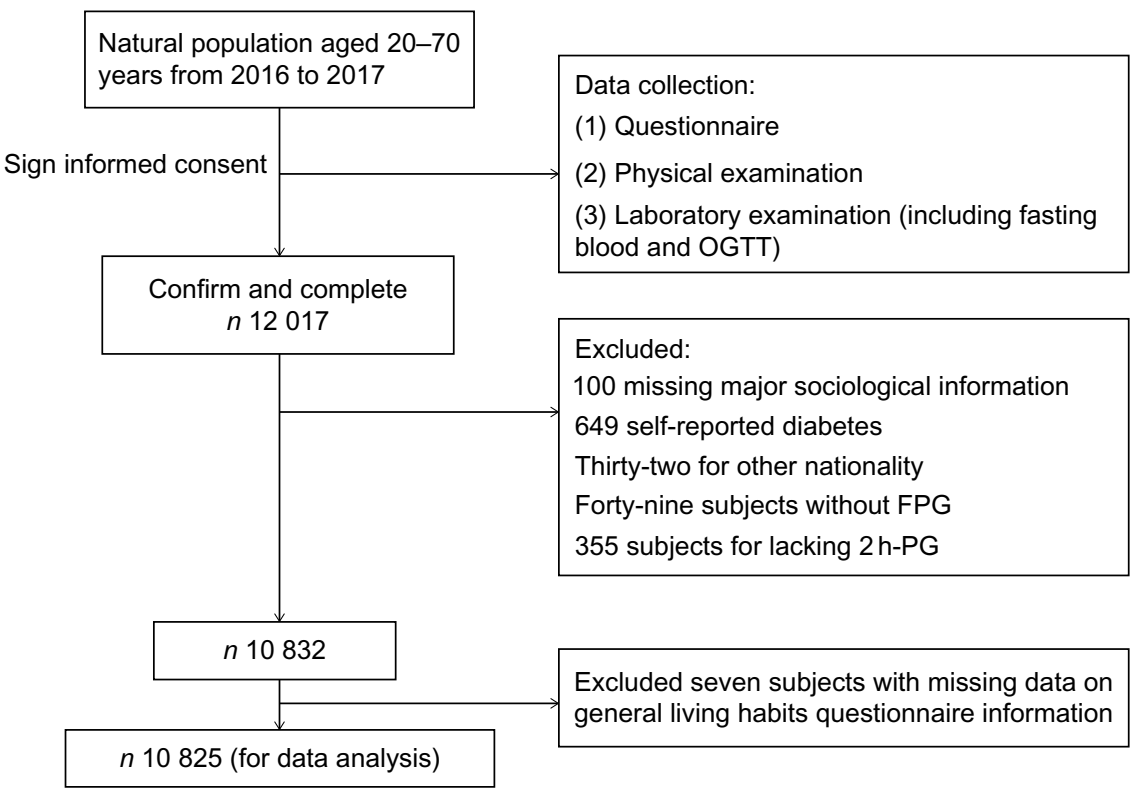

Fig. 1. Flow chart of the study population. OGTT, oral glucose tolerance test; FPG, fasting plasma glucose; PG, plasma glucose. 
The present study protocols were approved by the ethics committee of Zhongda Hospital, School of Medicine, Southeast University and other sub-centre hospitals involved, and written informed consent were signed from each participant before the study.

\section{Descriptive data collection}

A standardised questionnaire was used to collect demographic characteristics (sex, age, ethnicity, education level, annual household income and family history of diabetes) and lifestyle factors (diet habit, vegetable and fruit consumption, smoking, alcohol consumption, tea consumption, regular exercise participation and sedentary work) by trained doctors and nurses during the face-to-face interview.

\section{Clinical measurements}

All measurement instruments were conformed to the national metrological certification requirements, and all measurement methods met the industrial standard of the People's Republic of China human measurement methods (WS/t424-2013). Waist circumference was measured from the costal margin along the mid-axillary line to the iliac crest level at the horizontal plane. BMI was calculated as weight (kg) divided by height $(\mathrm{m})$ squared. Blood pressure was measured three times at 2-min intervals by a sphygmomanometer, and then the average of three measurements was taken. Fasting venous blood samples from the antecubital vein were obtained, and participants without T2DM conducted a 75-g oral glucose tolerance test performed by a trained staff. All blood samples stayed at room temperature for no more than $4 \mathrm{~h}$ and within the prescribed time limit for cold-chain transport $\left(2-8^{\circ} \mathrm{C}\right)$ to central laboratory for testing total cholesterol, TAG, HDL-cholesterol and LDLcholesterol and blood glucose.

\section{Assessments}

Newly diagnosed diabetes were defined as fasting plasma glucose $\geq 7.0 \mathrm{mmol} / \mathrm{l}$ or 2 -h plasma glucose $\geq 11.1 \mathrm{mmol} / \mathrm{l}$ according to diagnostic criteria published by the WHO in $1999^{(17)}$, without a history of diabetes. BMI was categorised for common obesity based on WHO suggestion: <25, 25-30 and $>30 \mathrm{~kg} / \mathrm{m}^{2}$. We define 'drinking tea every day' as daily tea drinkers, 'drinking tea at least once a week' as occasional tea drinkers, 'drinking tea at least once a month' as seldom tea drinkers. Smoking status and alcohol intake were assessed by using a structured questionnaire. Tea types in questionnaire are classified as green tea, white tea, oolong tea, black tea, dark tea, bottled tea beverage and other tea. To improve statistical power, white tea, oolong tea and bottled tea are combined with other types in the following analyses considering the small sample size.

\section{Statistical analyses}

First, 10825 participants' characteristics were assessed by diabetic status. The difference among newly diagnosed diabetes, pre-diabetes mellitus and non-diabetics was assessed with the $\chi^{2}$ and Kruskal-Wallis test, respectively. Second, hierarchical logistic regression was used to examine the association of tea consumption with newly diagnosed diabetes. Third, the interactive effect between tea consumption and sex/age/BMI on diabetes was explored by using Stata MP version 15.0. Statistical significance was defined as a $P$ value of $<0.05$ and based on two-sided probability.

\section{Results}

\section{Characteristics of study population}

Baseline characteristics of the 10825 participants according to diabetic status are presented in Table 1 . Compared with nondiabetic group, the proportion of newly diagnosed diabetes in men was higher $(41.6 \% v .29 \cdot 1 \%)$. Furthermore, these newly diagnosed diabetics were more likely to smoke cigarettes or drink alcohol, less likely to consume vegetables or fruit and had higher BMI, LDL, TAG, total cholesterol and systolic blood pressure. Of particular interest, the percentage of daily tea drinkers in diabetic patients was significantly lower than that of non-diabetic group ( $28.7 \% v .35 \cdot 4 \%)$.

\section{OR for type 2 diabetes mellitus according to tea-drinking status}

Table 2 shows the unadjusted prevalence of newly diagnosed diabetes: $9 \cdot 8,7 \cdot 4,7 \cdot 7$ and $7 \cdot 2 \%$ in the four groups. The crude OR of seldom tea drinkers, occasional tea drinkers and daily tea drinkers were 0.74 (95\% CI 0.59, 0.94), 0.77 (95\% CI 0.63, 0.94 ) and 0.72 (95\% CI 0.61, 0.85), respectively, compared with non-tea drinkers. In model 4 , after multiple adjustments, the prominent prevention impact of daily tea drinkers on T2DM declined slightly, but remained significant (OR 0.78; $95 \%$ CI $0 \cdot 65,0.94 ; P=0 \cdot 01$ ).

\section{Interactive effect of tea drinking with sex, age and BMI on type 2 diabetes mellitus}

To improve statistical power, we merged 'non-tea drinkers', 'seldom tea drinkers' and 'occasional tea drinkers' as 'non-daily tea drinkers'. As shown in Table 3, daily tea drinking was associated with a decreased risk of diabetes in female, elderly and obese subjects, with the adjusted OR of 0.68 (95\% CI $0.54,0.87), 0.77$ (95\% CI $0.64,0.93)$ and 0.66 (95\% CI 0.47 , $0.94)$, respectively. These results demonstrate a significant interactive effect of tea drinking with sex, age and BMI on T2DM $\quad\left(P_{\text {interaction } 1}=0.014, \quad P_{\text {interaction } 2}=0.043 \quad\right.$ and $P_{\text {interaction3 }}=0 \cdot 038$.

\section{The impact of tea types on type 2 diabetes mellitus}

To investigate the impact of tea types on the prevalence of undiagnosed T2DM, subgroup analyses were performed and are shown in Table 4. Those who consume dark tea (Pu'er) revealed the lowest newly diagnosed prevalence (5\%). After adjustments for covariates, including age, sex, BMI, systolic blood pressure, TAG, education level, family history of DM (yes or no), smoking status, alcohol consumption, vegetable consumption, food consumption, fruit consumption, sedentary work (yes or no) and regular physical exercise (yes or no), subjects consuming dark tea had a $46 \%$ decreased risk of T2DM, 
Table 1. Baseline characteristics of 10825 study participants according to the status of diabetes mellitus (Medians and interquartile ranges (IQR); numbers and percentages)

\begin{tabular}{|c|c|c|c|c|c|c|c|c|c|}
\hline \multirow[b]{2}{*}{ Variables } & \multicolumn{2}{|c|}{ Total population } & \multicolumn{2}{|c|}{ Non-diabetics } & \multicolumn{2}{|c|}{ Pre-diabetes* } & \multicolumn{2}{|c|}{ Newly diagnosed diabetes } & \multirow[b]{2}{*}{$P \dagger$} \\
\hline & Median & IQR & Median & IQR & Median & IQR & Median & IQR & \\
\hline$n$ & \multicolumn{2}{|c|}{10825} & \multicolumn{2}{|c|}{7401} & \multicolumn{2}{|c|}{2526} & \multicolumn{2}{|c|}{898} & \\
\hline$\%$ & & & & & & & & & \\
\hline \multicolumn{10}{|l|}{ Demographic data } \\
\hline Age & 51 & 40,60 & 48 & 37,57 & 55 & 47,63 & 58 & 51,64 & $<0.01$ \\
\hline \multicolumn{10}{|l|}{ Sex $(n$ and \%) } \\
\hline Male & 3400 & 31.4 & 2150 & $29 \cdot 1$ & 876 & 34.7 & 374 & $41 \cdot 6$ & $<0.01$ \\
\hline Female & 7425 & 68.6 & 5251 & $70 \cdot 9$ & 1650 & $65 \cdot 3$ & 524 & 58.4 & \\
\hline \multicolumn{10}{|l|}{ Educated level ( $n$ and \%) } \\
\hline Middle school or lower & 3993 & 36.9 & 2739 & $37 \cdot 0$ & 924 & $36 \cdot 6$ & 330 & $36 \cdot 7$ & 0.03 \\
\hline High school & 3514 & 32.5 & 2460 & 33.2 & 784 & 31.0 & 270 & $30 \cdot 1$ & \\
\hline College or higher & 3318 & $30 \cdot 7$ & 2202 & $29 \cdot 8$ & 818 & 32.4 & 298 & $33 \cdot 2$ & \\
\hline \multicolumn{10}{|c|}{ Annual household income ( $n$ and \%) } \\
\hline Less & 6010 & 55.5 & 4097 & 55.4 & 1417 & $56 \cdot 1$ & 496 & $55 \cdot 2$ & 0.90 \\
\hline Moderate & 2567 & $23 \cdot 7$ & 1773 & $24 \cdot 0$ & 585 & 23.2 & 209 & $23 \cdot 3$ & \\
\hline More & 2248 & $20 \cdot 8$ & 1531 & $20 \cdot 7$ & 524 & $20 \cdot 7$ & 193 & 21.5 & \\
\hline \multicolumn{10}{|c|}{ Family history of diabetes ( $n$ and \%) } \\
\hline Yes & 1444 & $13 \cdot 3$ & 884 & 11.9 & 379 & $15 \cdot 0$ & 181 & $20 \cdot 2$ & $<0.01$ \\
\hline No & 9381 & $86 \cdot 7$ & 6517 & $88 \cdot 1$ & 2147 & $85 \cdot 0$ & 717 & $79 \cdot 8$ & \\
\hline \multicolumn{10}{|l|}{ Lifestyle information } \\
\hline \multicolumn{10}{|l|}{ Smoking status ( $n$ and \%) } \\
\hline Current smokers & 1526 & $14 \cdot 1$ & 970 & $13 \cdot 1$ & 391 & $15 \cdot 5$ & 165 & 18.4 & $<0.01$ \\
\hline Ex-smokers & 324 & 3.0 & 177 & 2.4 & 113 & 4.5 & 34 & $3 \cdot 8$ & \\
\hline Never smokers & 8975 & 82.9 & 6254 & 84.5 & 2022 & $80 \cdot 0$ & 699 & $77 \cdot 8$ & \\
\hline \multicolumn{10}{|c|}{ Alcohol consumption status ( $n$ and \%) } \\
\hline Current drinkers & 2213 & 20.4 & 1393 & $18 \cdot 8$ & 566 & $22 \cdot 4$ & 254 & $28 \cdot 3$ & $<0.01$ \\
\hline Ex-drinkers & 423 & 3.9 & 260 & 3.5 & 122 & 4.8 & 41 & 4.6 & \\
\hline Never drinkers & 8189 & $75 \cdot 6$ & 5748 & $77 \cdot 7$ & 1838 & $72 \cdot 8$ & 603 & $67 \cdot 1$ & \\
\hline \multicolumn{10}{|c|}{ Food consuming amount ( $n$ and \%) } \\
\hline Excess & 1201 & $11 \cdot 1$ & 786 & $10 \cdot 6$ & 284 & 11.2 & 131 & $14 \cdot 6$ & $<0.01$ \\
\hline Normal & 8983 & $83 \cdot 0$ & 6206 & 83.9 & 2079 & $82 \cdot 3$ & 698 & $77 \cdot 7$ & \\
\hline Less & 641 & 5.9 & 409 & 5.5 & 163 & 6.5 & 69 & $7 \cdot 7$ & \\
\hline \multicolumn{10}{|c|}{ Vegetable consuming amount ( $n$ and \%) } \\
\hline Excess & 3222 & 29.8 & 2190 & $29 \cdot 6$ & 759 & $30 \cdot 0$ & 273 & $30 \cdot 4$ & 0.01 \\
\hline Normal & 7009 & 64.7 & 4823 & $65 \cdot 2$ & 1633 & 64.6 & 553 & 61.6 & \\
\hline Less & 594 & 5.5 & 388 & $5 \cdot 2$ & 134 & $5 \cdot 3$ & 72 & 8.0 & \\
\hline \multicolumn{10}{|c|}{ Fruit consuming amount ( $n$ and \%) } \\
\hline Excess & 1678 & $15 \cdot 5$ & 1198 & $16 \cdot 2$ & 366 & 14.5 & 114 & $12 \cdot 7$ & $<0.01$ \\
\hline Normal & 6701 & 61.9 & 4657 & 62.9 & 1541 & 61.0 & 503 & $56 \cdot 0$ & \\
\hline & 2446 & $22 \cdot 6$ & 1546 & $20 \cdot 9$ & 619 & 24.5 & 281 & $31 \cdot 3$ & \\
\hline \multicolumn{10}{|l|}{ Sedentary work ( $n$ and \%) } \\
\hline Yes & 3234 & 29.9 & 2157 & $29 \cdot 1$ & 796 & 31.5 & 281 & 31.3 & 0.14 \\
\hline No & 7058 & $65 \cdot 2$ & 4885 & $66 \cdot 0$ & 1599 & 63.3 & 574 & 63.9 & \\
\hline Unclear & 533 & 4.9 & 359 & 4.9 & 131 & $5 \cdot 2$ & 43 & 4.8 & \\
\hline
\end{tabular}




\section{N British Journal of Nutrition}

Table 1. (Continued)

\begin{tabular}{|c|c|c|c|c|c|c|c|c|c|}
\hline \multirow[b]{2}{*}{ Variables } & \multicolumn{2}{|c|}{ Total population } & \multicolumn{2}{|c|}{ Non-diabetics } & \multicolumn{2}{|c|}{ Pre-diabetes* } & \multicolumn{2}{|c|}{ Newly diagnosed diabetes } & \multirow[b]{2}{*}{$P \dagger$} \\
\hline & Median & IQR & Median & IQR & Median & IQR & Median & IQR & \\
\hline \multicolumn{10}{|c|}{ Physical exercise regularly ( $n$ and \%) } \\
\hline Yes & 4192 & $38 \cdot 7$ & 2780 & 37.6 & 1026 & $40 \cdot 6$ & 386 & $43 \cdot 0$ & \multirow[t]{2}{*}{0.01} \\
\hline No & 6633 & $61 \cdot 3$ & 4621 & $62 \cdot 4$ & 1500 & 59.4 & 512 & $57 \cdot 0$ & \\
\hline \multicolumn{10}{|l|}{ Tea-drinking status ( $n$ and \%) } \\
\hline Never drink tea & 4118 & 38.0 & 2602 & $35 \cdot 2$ & 1114 & $44 \cdot 1$ & 402 & 44.8 & \multirow[t]{4}{*}{$<0.01$} \\
\hline Tea-drinking seldom & 1254 & 11.6 & 881 & 11.9 & 280 & $11 \cdot 1$ & 93 & $10 \cdot 4$ & \\
\hline Tea-drinking occasionally & 1887 & $17 \cdot 4$ & 1301 & $17 \cdot 6$ & 441 & 17.5 & 145 & $16 \cdot 1$ & \\
\hline Tea-drinking daily & 3566 & $32 \cdot 9$ & 2617 & $35 \cdot 4$ & 691 & $27 \cdot 4$ & 258 & $28 \cdot 7$ & \\
\hline \multicolumn{10}{|l|}{ Laboratory examination } \\
\hline BMI $\left(\mathrm{kg} / \mathrm{m}^{2}\right)$ & 24 & 22,27 & 24 & 22, 27 & 25 & 23,28 & 26 & 24,29 & $<0.01$ \\
\hline TAG (mmol/l) & $1 \cdot 18$ & $0.81,1.78$ & 1.07 & $0.75,1.56$ & 1.42 & $0.96,2 \cdot 13$ & 1.72 & $1 \cdot 14,2 \cdot 68$ & $<0.01$ \\
\hline $\mathrm{TC}(\mathrm{mmol} / \mathrm{l})$ & 5.07 & $4 \cdot 35,5 \cdot 87$ & 4.93 & $4 \cdot 24,5 \cdot 71$ & $5 \cdot 33$ & $4.59,6.08$ & 5.52 & $4.76,6 \cdot 33$ & $<0.01$ \\
\hline $\mathrm{HDL}(\mathrm{mmol} / \mathrm{l})$ & 1.54 & $1 \cdot 30,1 \cdot 81$ & 1.56 & $1.33,1.82$ & 1.51 & $1.27,1 \cdot 78$ & 1.47 & $1 \cdot 25,1 \cdot 73$ & $<0.01$ \\
\hline LDL (mmol/l) & 2.90 & $2 \cdot 36,3.49$ & 2.79 & $2 \cdot 28,3 \cdot 37$ & 3.08 & $2.55,3.64$ & $3 \cdot 21$ & $2.68,3.79$ & $<0.01$ \\
\hline $\mathrm{DBP}(\mathrm{mmHg})$ & 80 & 72,88 & 78 & 70,86 & 83 & 76,91 & 86 & 78,95 & $<0.01$ \\
\hline $\mathrm{SBP}(\mathrm{mmHg})$ & 127 & 115,143 & 124 & 112,138 & 134 & 122,150 & 142 & 129,156 & $<0.01$ \\
\hline
\end{tabular}

TC, total cholesterol; DBP, diastolic blood pressure; SBP, systolic blood pressure.

Pre-diabetes: impaired fasting glucose (6.1-7.0 mmo//), impaired glucose tolerance $(7.8-11.1 \mathrm{mmol} / \mathrm{l})$ or both exist, according to diagnostic criteria published by the WHO in $1999^{(17)}$

† For continuous data with skewed distribution, the data are presented as medians and IQR and analysed by the Kruskal-Wallis test. Categorical data are presented as $n$ and $\%$ and analysed by the $\chi^{2}$ test. Significant level, $\alpha=0.05$.

Table 2. Newly diagnosed incidence rates and risk for type 2 diabetes mellitus according to tea drinking status

(Odds ratios and $95 \%$ confidence intervals)

\begin{tabular}{|c|c|c|c|c|c|c|c|c|}
\hline & \multirow[b]{2}{*}{ Never drink tea ( $n$ 4118): OR } & \multicolumn{2}{|c|}{$\begin{array}{l}\text { Tea-drinking seldom } \\
(n \text { 1254) }\end{array}$} & \multicolumn{2}{|c|}{$\begin{array}{l}\text { Tea-drinking occasionally } \\
\qquad(n \text { 1887) }\end{array}$} & \multicolumn{2}{|c|}{ Tea-drinking daily ( $n$ 3566) } & \multirow[b]{2}{*}{$P_{\text {for trend }}$} \\
\hline & & OR & $95 \% \mathrm{Cl}$ & OR & $95 \% \mathrm{Cl}$ & OR & $95 \% \mathrm{Cl}$ & \\
\hline Cases & 402 & & & & & & & \\
\hline Rate (\%) & 9.8 & & & & & & & \\
\hline Model $1^{*}$ & 1 (Ref) & 0.74 & $0.59,0.94$ & 0.77 & $0.63,0.94$ & 0.72 & $0.61,0.85$ & $<0.001$ \\
\hline Model $2 \dagger$ & 1 (Ref) & 0.86 & $0.67,1.09$ & 0.87 & $0.71,1.07$ & 0.68 & $0.57,0.81$ & $<0.001$ \\
\hline Model 3ł & 1 (Ref) & 0.80 & $0.62,1.02$ & 0.82 & $0.67,1.02$ & 0.73 & $0.61,0.87$ & 0.006 \\
\hline Model $4 \S$ & 1 (Ref) & 0.83 & $0.65,1.07$ & 0.86 & $0.69,1.06$ & 0.78 & $0.65,0.94$ & 0.010 \\
\hline
\end{tabular}

Ref, reference.

* Model 4: adjusted for all factors in model 3 plus smoking status, alcohol consumption status, vegetable consumption, food consumption, fruit consumption, sedentary work (yes or no), physical exercise regularly (yes or no).

† Model 1: crude.

$\ddagger$ Model 2: adjusted for factors in model 1 plus age, sex and BMI.

$\S$ Model 3: adjusted for all factors in model 2 plus systolic blood pressure, TAG, education level, family history of diabetes mellitus (yes or no). 
Table 3. Interactive effect of tea consumption on the risk of diabetes (Odds ratios and $95 \%$ confidence intervals)

\begin{tabular}{|c|c|c|c|c|c|c|c|}
\hline \multirow[b]{2}{*}{ Variables } & \multicolumn{2}{|c|}{$\begin{array}{l}\text { Tea-drinking } \\
\text { non-daily }\end{array}$} & \multicolumn{2}{|c|}{ Tea-drinking daily } & \multicolumn{3}{|c|}{ Effect of tea* } \\
\hline & DM & $\%$ & DM & $\%$ & OR & $95 \% \mathrm{Cl}$ & $P^{\star}$ \\
\hline \multicolumn{8}{|l|}{ Sex } \\
\hline Male & 220 & $11 \cdot 2$ & 154 & $10 \cdot 8$ & 1.03 & $0.82,1.31$ & 0.794 \\
\hline Female & 420 & $7 \cdot 9$ & 104 & 4.9 & 0.68 & $0.54,0.87$ & 0.002 \\
\hline $\begin{array}{r}P_{\text {interaction } 1} \\
\text { Age (years) }\end{array}$ & & & & & & & 0.014 \\
\hline $20-44$ & 60 & $2 \cdot 8$ & 52 & $3 \cdot 2$ & 1.21 & $0.81,1.78$ & 0.356 \\
\hline $45-70$ & 580 & $11 \cdot 3$ & 206 & 10.5 & 0.77 & $0.64,0.93$ & 0.005 \\
\hline $\begin{array}{r}P_{\text {interaction2 }} \\
\text { BMl }\left(\mathrm{kg} / \mathrm{m}^{2}\right)\end{array}$ & & & & & & & 0.043 \\
\hline$\leq 30$ & 545 & $8 \cdot 1$ & 189 & $6 \cdot 4$ & 1.00 & $0.84,1 \cdot 21$ & 0.837 \\
\hline$>30$ & 95 & $19 \cdot 1$ & 69 & $11 \cdot 1$ & 0.66 & $0.47,0.94$ & 0.018 \\
\hline$P_{\text {interaction3 }}$ & & & & & & & 0.038 \\
\hline
\end{tabular}

* Adjusted for other confounding factors other than interaction variables.

Table 4. Newly diagnosed incidence rates and risk for type 2 diabetes mellitus according to tea types (Odds ratios and $95 \%$ confidence intervals)

\begin{tabular}{|c|c|c|c|c|c|c|c|c|c|}
\hline & \multirow[b]{2}{*}{$n$} & \multirow[b]{2}{*}{$\%$} & \multirow[b]{2}{*}{ Cases } & \multirow[b]{2}{*}{ Prevalence } & \multicolumn{2}{|c|}{$\begin{array}{l}\text { Adjusted for sex, age } \\
\text { and BMI }\end{array}$} & \multicolumn{2}{|c|}{$\begin{array}{l}\text { Multivariate } \\
\text { adjustment }^{\star}\end{array}$} & \multirow[b]{2}{*}{$P^{\star}$} \\
\hline & & & & & OR & $95 \% \mathrm{Cl}$ & OR & $95 \% \mathrm{Cl}$ & \\
\hline Never drink tea & 4118 & 380 & 402 & $9 \cdot 8$ & 1 & Ref & 1 & Ref & 0.001 \\
\hline Green tea & 1310 & 121 & 125 & 9.5 & 0.96 & $0.77,1.20$ & 0.93 & $0.74,1.17$ & 0.556 \\
\hline Black tea & 1443 & 133 & 84 & $5 \cdot 8$ & 0.65 & $0.50,0.84$ & 0.85 & $0.65,1.11$ & 0.226 \\
\hline Dark tea & 1543 & 143 & 77 & $5 \cdot 0$ & 0.46 & $0.35,0.60$ & 0.54 & $0.41,0.72$ & $<0.001$ \\
\hline Other tea & 1307 & 121 & 116 & 8.9 & 0.97 & $0.77,1.21$ & 0.96 & $0.76,1 \cdot 21$ & 0.702 \\
\hline More than one type & 1104 & 102 & 94 & 8.5 & 0.80 & $0.63,1.02$ & 0.79 & $0.61,1.02$ & 0.072 \\
\hline
\end{tabular}

Ref, reference.

${ }^{*}$ Adjusted for sex, age, BMl, systolic blood pressure, TAG, education level, family history of diabetes mellitus (yes or no), smoking status, alcohol consumption status, vegetable consumption, food consuming, fruit, sedentary work (yes or no), physical exercise regularly (yes or no).

compared with non-tea drinkers. However, subjects drinking green tea and black tea did not show significant reduction, with adjusted OR of $0.93(0 \cdot 74,1 \cdot 17)$ and $0.85(0.65,1 \cdot 11)$, respectively.

\section{Discussion}

According to our knowledge, this is the first national observational study addressing the relationship of tea-drinking status and various tea types with T2DM in China. In our study, about $62 \%$ of Chinese is drinking tea, mainly dark tea, black tea and green tea, accounting for $14.3,13.3$ and $12 \cdot 1 \%$, respectively. Moreover, $32.9 \%$ of subjects have the habit of drinking tea daily. It is fully confirmed that it is necessary to study the impact of tea drinking on the risk of diabetes in the Chinese population. According to the research, the total food consumption, alcohol consumption and physical exercise regularly of daily tea drinkers were lower than those of non-daily tea drinkers; however, vegetable consumption and fruit consumption of them were more than those of non-daily tea drinkers in men and women $(P<0 \cdot 05)$ (data not shown). It could be speculated that dietary and living habits of tea drinkers daily are significantly different from those of nondaily tea drinkers in China. The interaction between diet habits and tea drinking, which has been not reported by previous researchers, requires further exploration in the future. Furthermore, the diet and living habits have been adjusted to increase the credibility of those results in these following results.

Our results observed a negative association between tea consumption and risk of T2DM. These findings are inconsistent with several previous studies ${ }^{(15,18,19)}$, which observed a higher risk of T2DM in tea consumers, but consistent with several previous studies $^{(13,20-22)}$ and meta-analyses ${ }^{(23,24)}$, in which a negative association was discovered. This discrepancy could be due to subjects, types or frequency of tea drinking. Additionally, a positive association between pesticides and T2DM has also been found in several studies ${ }^{(25-27)}$. Liu et al.'s study in Shanghai also proposed that more pesticide exposures among tea drinkers may be associated with an increased risk of $\mathrm{T}_{2} \mathrm{DM}^{(18)}$. Besides, drinking tea daily is more beneficial to prevent T2DM than seldom or occasional tea drinking in this research. Our result is in accordance with a meta-analysis ${ }^{(23)}$ in which drinking more than three cups of tea per d was associated with a lower risk of T2DM. Additionally, Jing et al. ${ }^{(24)}$ also found that consuming more than four cups per d may reduce the risk of T2DM by approximately $20 \%$ (risk ratio 0.80; $95 \%$ CI 0.70, 0.93). Furthermore, experimental studies have discovered that several bioactive 
components, including catechins, gallic acid, polysaccharides and epigallocatechin-3-gallate, within tea show antioxidant $\operatorname{activity}^{(28,29)}$ and improve insulin sensitivity ${ }^{(30)}$. The polysaccharides extracted from fully fermented black tea and dark tea contain a higher percentage of low molecular weight portions (antioxidants) than those extracted from green tea and oolong tea, resulting in more benefits to $\mathrm{T}_{2} \mathrm{DM}^{(9)}$. According to Bhupathiraju et al., the effect of tea on diabetes might be attributed to caffeine. The study demonstrated that caffeinated tea could lower the risk of T2DM among women ${ }^{(31)}$. To sum up, these effects and mechanisms mentioned above are discrepant, possibly caused by different subjects, various tea types or tea drinking frequencies in previous researches; therefore, further evidence of the impact of tea drinking on Chinese is required in the future.

In our study, the negative correlation of daily tea drinking with the risk of T2DM is likely influenced by sex, age and BMI, which was determined by the significant interaction effect. Our results suggest that daily tea drinking may diminish the risk of diabetes in female by $32 \%$, elderly by $23 \%$ and obese subjects by $34 \%$. Yang et al.'s finding also demonstrated that drinking tea was associated with a reduced risk of diabetes in women and no significant effect in men was found ${ }^{(23)}$; this disparity may be related to more men smoking than women, and a linear positive correlation between the amount of smoking and the risk of diabetes has previously been acknowledged ${ }^{(32)}$. Additionally, the significant interaction effect between daily tea drinking and smoking is also found ( $P_{\text {interaction }}=0.015$ ) (data not shown), which demonstrates that daily tea drinking is associated with lowering the risk of diabetes in non-smokers, not significant effective on smokers. Therefore, smoking (mostly male) would counteract the protective effect of daily tea drinking on diabetes, which is consistent with the above result. Moreover, in our study, daily tea consumption was not significantly associated with weight loss, which is supported by Greenberg et al.'s cohort study ${ }^{(33)}$. Therefore, we can speculate that the negative association between drinking tea daily and the risk of T2DM in obese may be achieved through increasing insulin sensitivity of obese patients, but more research on the mechanisms is needed in the future. In addition, we showed a decreased risk of diabetes in the elderly, which coincide with that of the Singapore Chinese Health Study ${ }^{(13)}$. This difference could be due to young people's preference for bottled or sugary beverage, which has been demonstrated to increase the risk of $\mathrm{T}_{2} \mathrm{DM}^{(34)}$. In our study, the proportion of young people drinking bottled tea $(2 \cdot 3 \%)$ was three times that of the elderly $(0.7 \%)$. However, because of the small sample of subjects who drink bottled tea, larger sample studies are needed to verify this speculation. In addition, the shorter duration of tea drinking by young people may also be a factor of the results, long-term follow-up studies are necessary.

As for tea types, the diabetes-related research on rats ${ }^{(35)}$ has suggested potential benefits of green and black teas in insulin and glucose metabolism. Mahmoud's cohort work discovered that administrating three cups per $\mathrm{d}$ of black tea can ameliorate the progression of T2DM in adults ${ }^{(36)}$. The Singapore Chinese study had a suggestive $14 \%$ (risk ratio $0.86 ; 95 \% \mathrm{CI}$ $0.74,1.00)$ reduction in risk of diabetes within participants drinking black tea more than one cup per d compared with non-consumers, but there was no association observed concerning green tea ${ }^{(13)}$. Additionally, daily consumption of black tea has been confirmed to increase the level of plasma antioxidants such as glutathione and decrease serum C-reactive protein level in diabetic populations ${ }^{(37,38)}$. Moreover, Deng et al. ${ }^{(20)}$ observed, in vitro and in vivo, that Pu'er (dark tea) could suppress blood sugar in diabetes by inhibiting $\alpha$-glucosidase activity. In our study, both black tea and dark tea were related to reducing the risk of diabetes after adjusting for sex, age and BMI; however, after adjusting for other confounding factors, the effect of dark tea remained significant (OR 0.54; $95 \% \mathrm{CI}$ $0 \cdot 41,0 \cdot 72 ; P<0 \cdot 001$ ), while no significance was observed in black and green tea, consistent with the study in Japan ${ }^{(14)}$.

To our knowledge, as fully fermented tea, dark tea is a unique tea type in China and population-based research about dark tea is lacking. Our results showed that whole fermented tea, especially dark tea, is associated with a reduced risk of diabetes. Nevertheless, the disagreements with some of the previous studies about green or black tea could most likely be influenced by research population (ethnics), tea preparation methods, concentration of tea, level of pesticides exposure in tea or other unadjusted incorporated lifestyles.

These findings convey a potential high significance for public health because tea is the second most consumed beverages worldwide after water. Though China has a long history of tea drinking, former cohort studies on this topic had been limited to western populations. The highlights of the present study are summarised as follows: (1) The present study includes a large sample population from eight different sites in five areas in China, and the questionnaire information was comprehensive. (2) This is the first time to explore the interaction between sex, age and BMI with daily tea drinking on diabetes risk, and the result plays an instructive role in personalised prevention of diabetes. (3) The types of tea are classified in detail, whereas previous studies were mostly limited to green tea, black tea or oolong tea, solely. (4) The oral glucose tolerance test was tested for diagnosis of diabetes in the present study, and bias related to self-reports was avoided. (5) The present study did not contain already diagnosed patients, whose lifestyles had been influenced by medical intervention (data not shown).

However, several limitations of the present study should be considered: (1) This is a cross-sectional study that can only explain correlation and not causality. (2) The data related to lifestyles were obtained from the self-reported questionnaire, likely containing memory and self-reported bias. (3) The category of tea consumption status was not quantified (specific to frequency), possibly resulting in understanding bias. (4) Other unknown confounders potentially exist, such as milk, sweet foods and beverages consumption status, despite adjustments for multiple confounding factors. For example, Kazakhs and Uyghurs in China drink salty tea with mare milk at meal time, especially breakfast. It is not clear whether the effect of tea is affected by milk or salt, and the interaction of different micronutrients also needs to be clarified in the future. (5) The results of the present study are limited to the Chinese population and may be ineffective against other ethnic groups, such as Caucasians, with characteristic genes or habits.

In summary, our study suggests that daily tea consumption is negatively associated with the risk of T2DM among female, 
elderly and obese people. Furthermore, consuming dark tea in particular showed the greatest association with reduced risk of diabetes in the Chinese population. Further cohorts studies should be conducted to elucidate the effect of tea on T2DM.

\section{Acknowledgements}

We thank all the participants, cooperative hospitals and investigators who contributed their precious time in this research.

The present study was supported by the National Key R\&D Program of China (no. 2016YFC1305700), the National Key Scientific Instrument and Equipment Development Project of China (no. 51627808) and the grants from the Excellence Project of Southeast University (no. SJCX17_0072).

Z. S., Y. C. and W. L. were responsible for the study design. Y. C., W. L., S. Q. and B. W. were responsible for data collection, data analysis, interpretation and writing of the manuscript. Z. S., S. H., X. X., C. V., X. W., X. N., Q. C., Q. W., P. T., L. Z., S. Y., K. L., J. C., H. W., X. W., X. W., J. L., M. C. and Z. W. assisted with the data collection and manuscript modification. All authors have read the manuscript critically and approved the submitted version.

The authors declare no conflicts of interest of any commercial or financial relationships.

\section{References}

1. Wang L, Gao P, Zhang M, et al. (2017) Prevalence and ethnic pattern of diabetes and prediabetes in China in 2013. JAMA 317, 2515.

2. Farag YM \& Gaballa MR (2011) Diabesity: an overview of a rising epidemic. Nephrology, dialysis, transplantation: official publication of the European dialysis and transplant association. Eur Renal Assoc 26, 28-35.

3. Kolb H \& Martin S (2017) Environmental/lifestyle factors in the pathogenesis and prevention of type 2 diabetes. BMCMed 15, 131.

4. Pan XR, Li GW, Hu YH, et al. (1997) Effect of diet and exercise in preventing NIDDM in people with impaired glucose tolerance. Diab Care 20, 537.

5. Golozar A, Khademi H, Kamangar F, et al. (2011) Diabetes mellitus and its correlates in an Iranian adult population. PLOS ONE 6, e26725.

6. Yajima H (2014) Prevention of diet-induced obesity by dietary polyphenols derived from Nelumbo nucifera and black tea. Polyphenols Hum Health Dis 15, 135-142.

7. Chan EWC, Soh EY, Pei PT, et al. (2011) Antioxidant and antibacterial properties of green, black, and herbal teas of Camellia sinensis. Pharmacogn Res 3, 266-272.

8. Sanlier N, Gokcen BB \& Altug M (2018) Tea consumption and disease correlations. Trends Food Sci Technol 78, 95-106.

9. Fu QY, Li QS, Lin XM, et al. (2017) Antidiabetic effects of tea. Molecules 22, 849.

10. Gondoin A, Grussu D, Stewart D, et al. (2010) White and green tea polyphenols inhibit pancreatic lipase in vitro. Food Res Int 43, 1537-1544.

11. Mao JT (2013) Chapter 3. White tea: the plants, processing, manufacturing, and potential health benefits. In Tea in Health and Disease Prevention, pp. 33-40 [VR Preedy, editor]. London: Academic Press.

12. Grosso G, Stepaniak U, Micek A, et al. (2015) Association of daily coffee and tea consumption and metabolic syndrome: results from the Polish arm of the HAPIEE study. Eur J Nutr 54, 1129-1137.
13. Odegaard AO, Pereira MA, Koh WP, et al. (2008) Coffee, tea, and incident type 2 diabetes: the Singapore Chinese health study. Am J Clin Nutr 88, 979-985.

14. Oba S, Nagata C, Nakamura K, et al. (2010) Consumption of coffee, green tea, oolong tea, black tea, chocolate snacks and the caffeine content in relation to risk of diabetes in Japanese men and women. Br J Nutr 103, 453-459.

15. Siddiqui FJ, Avan BI, Mahmud S, et al. (2015) Uncontrolled diabetes mellitus: prevalence and risk factors among people with type 2 diabetes mellitus in an Urban District of Karachi, Pakistan. Diab Res Clin Prac 107, 148-156.

16. Li W, Xie B, Qiu S, et al. (2018) Non-lab and semi-lab algorithms for screening undiagnosed diabetes: a cross-sectional study. EBioMed 35, 307-316.

17. Drouin P, Blickle JF \& Charbonnel B (1999) Diagnosis and classification of diabetes mellitus: the new criteria. Diab Metabol 25, 72-83.

18. Liu X, Xu W, Cai H, et al. (2018) Green tea consumption and risk of type 2 diabetes in Chinese adults: the Shanghai Women's Health Study and the Shanghai Men's Health Study. Int J Epidemiol 47, 1887-1896.

19. Hayashino Y, Fukuhara S, Okamura T, et al. (2011) High oolong tea consumption predicts future risk of diabetes among Japanese male workers: a prospective cohort study. Diab Med 28, 805-810.

20. Deng YT, Lin-Shiau SY, Shyur LF, et al. (2015) Pu-erh tea polysaccharides decrease blood sugar by inhibition of $\alpha$-glucosidase activity in vitro and in mice. Food Func $\mathbf{6}$, 1539-1546.

21. Striegel L, Kang B, Pilkenton SJ, et al. (2015) Effect of black tea and black tea pomace polyphenols on $\alpha$-glucosidase and $\alpha$-amylase inhibition, relevant to type 2 diabetes prevention. Front Nutr 2, 3

22. Yao Z, Gu Y, Zhang Q, et al. (2019) Estimated daily quercetin intake and association with the prevalence of type 2 diabetes mellitus in Chinese adults. Eur J Nutr 58, 819-830.

23. Yang W-S, Wang W-Y, Fan W-Y, et al. (2013) Tea consumption and risk of type 2 diabetes: a dose-response meta-analysis of cohort studies. Br J Nutr 111, 1329-1339.

24. Jing Y, Han G, Hu Y, et al. (2009) Tea consumption and risk of type 2 diabetes: a meta-analysis of cohort studies. J General Internal Med 24, 557-562.

25. Montgomery MP, Kamel F, Saldana TM, et al. (2008) Incident diabetes and pesticide exposure among licensed pesticide applicators: Agricultural Health Study, 1993-2003. Am J Epidemiol 167, 1235-1246.

26. Han K, Hwang E \& Park J (2016) Excessive consumption of green tea as a risk factor for periodontal disease among Korean adults. Nutrients $\mathbf{8}, 408$.

27. Son HK, Kim SA \& Kang JH, et al. (2010) Strong associations between low-dose organochlorine pesticides and type 2 diabetes in Korea. Environ Int 36, 410-414.

28. Kongpichitchoke T, Chiu MT, Huang TC, et al. (2016) Gallic acid content in Taiwanese teas at different degrees of fermentation and its antioxidant activity by inhibiting PKC $\delta$ activation: in vitro and in silico studies. Molecules 21, 1346.

29. Chen H, Qu Z, Fu L, et al. (2010) Physicochemical properties and antioxidant capacity of 3 polysaccharides from green tea, oolong tea, and black tea. J Food Sci 74, C469-C474.

30. Zheng XX, Xu YL, Li SH, et al. (2013) Effects of green tea catechins with or without caffeine on glycemic control in adults: a meta-analysis of randomized controlled trials. Am J Clin Nutr 97, 750-762.

31. Bhupathiraju SN, Pan A, Malik VS, et al. (2013) Caffeinated and caffeine-free beverages and risk of type 2 diabetes. Am J Clin Nutr 97, 155-166. 
32. Akter S, Goto A \& Mizoue T (2017) Smoking and the risk of type 2 diabetes in Japan: a systematic review and meta-analysis. J Epidemiol 27, 553-561.

33. Greenberg JA, Axen KV, Schnoll R, et al. (2005) Coffee, tea and diabetes: the role of weight loss and caffeine. Int JObes 29, 1121.

34. O'Connor L, Imamura F, Lentjes MAH, et al. (2015) Prospective associations and population impact of sweet beverage intake and type 2 diabetes, and effects of substitutions with alternative beverages. Diabetologia 58, 1474-1483.

35. Wu LY, Juan CC, Ho LT, et al. (2004) Effect of green tea supplementation on insulin sensitivity in Sprague-Dawley rats. J Agric Food Chem 52, 643-648.
36. Mahmoud F, Haines D, Al-Ozairi E, et al. (2016) Effect of black tea consumption on intracellular cytokines, regulatory $\mathrm{T}$ cells and metabolic biomarkers in type 2 diabetes patients. Phytother Res 30, 454-462.

37. Ãz H, Luna C \& Estévez M (2016) Redox chemistry of the molecular interactions between tea catechins and human serum proteins under simulated hyperglycemic conditions. Food Func 7, 1390-1400.

38. Neyestani TR, Shariatzade N, Kalayi A, et al. (2010) Regular daily intake of black tea improves oxidative stress biomarkers and decreases serum C-reactive protein levels in type 2 diabetic patients. Ann Nutr Metabol 57, 40-49. 